\title{
Corrosión microbiológica en aceros de bajo carbono
}

\section{Microbiological Corrosion in Low Carbon Steels}

\author{
O. Medina-Custodio \\ Unidad de Investigación y Asistencia Técnica en Materiales (UDIATEM), DIMEI, Facultad de Ingeniería, UNAM. \\ E-mail:medina_1979@yahoo.com \\ A. Ortiz-Prado \\ Unidad de Investigación y Asistencia Técnica en Materiales (UDIATEM), DIMEI, Facultad de Ingeniería, UNAM. \\ E-mail:armandoo@servidor.unam.mx \\ V.H. Jacobo-Armendáriz \\ Unidad de Investigación y Asistencia Técnica en Materiales (UDIATEM), DIMEI, Facultad de Ingeniería, UNAM. \\ E-mail:vjacobo@dgapa.unam.mx \\ R. Schouwenaars-Franssens \\ Unidad de Investigación y Asistencia Técnica en Materiales (UDIATEM), DIMEI, Facultad de Ingeniería, UNAM. \\ E-mail:raf_schouweenaars@yahoo.com
}

(Recibido: mayo de 2006; reevaluado: mayo de 2007; aceptado: agosto de 2007)

\section{Resumen}

La corrosión microbiológica es un tipo común de deterioro que afecta diversas industrias, una de ellas es la petrolera en la que se estiman que el $20 \%$ o $30 \%$ de fallas en las tuberías de trasporte de hidrocarburos es favorecida por microorganismos. Las reacciones químicas que sustentan éstos, generan transferencia de iones, lo que justifica el empleo de técnicas electroquímicas para su análisis. En este trabajo, se estudiaron probetas de acero de bajo carbono SAE 1018, sumergidas en un medio nutritivo rico en cloruros en presencia y ausencia de tres diferentes cargas microbianas, en tiempos de exposición de 48 horas y 28 días. Se realizaron ensayos de resistencia a la polarización (Rp) y espectroscopia de impedancia electroquímica (EIS) para determinar el efecto corrosivo de los diferentes sistemas. Los resultados muestran que el medio abiótico causa el mayor efecto corrosivo, lo que indica un efecto protector de los microorganismos al metal, contradiciendo la hipótesis inicialmente propuesta. La observación de las superficies en el microscopio electrónico de barrido (MEB) apoyan lo obtenido por las técnicas electroquímicas. Para explicar la disminución de la corrosividad del medio en presencia de microorganismos se propone un posible mecanismo de polarización catódica.

Descriptores: Corrosión microbiológica, acero de bajo carbono, técnicas electroquímicas.
Abstract
The Microbiologically Induced Corrosion affects several industries, such as oil industry where it is estimated that $20 \%$ to $30 \%$ pipes failures are related with microorganism. The chemical reactions generate ions transfer, this validate the use of electrochemical techni- ques for its analysis. Coupons submerged in a nutritional medium with presence and ab- sence of three different microorganisms during two periods, 48 hours and 28 days were studied. Polarization resistance (Rp) and Electrochemical Impedance Spectroscopy (EIS) techniques were applied to determine the corrosivity of the systems. The results show a 
greater corrosive effect of abiotic system, this indicates a microorganisms protection effect to the metal, opposite to the first hypothesis. This result was ratified observing surfaces coupons by using Scanning Electron Microscopy (SEM) technique. A possible mechanism based on Evans - Tafel graph is proposed to explain inhibitor microorganism effect.

Keywords: Low carbon steel, Microbiologically Induced Corrosion (MIC), electrochemical techniques.

\section{Introducción}

Un tipo de deterioro al que se le ha dado importancia en las últimas décadas es la corrosión microbiológica, también conocida como corrosión inducida microbiologicamente o biocorrosión; la cual se define como un proceso electroquímico donde la participación de los microorganismos es capaz de iniciar, facilitar o acelerar una reacción de corrosión sin modificar su naturaleza electroquímica (Borenstein, 1994).

Este tipo de corrosión afecta industrias como la eléctrica, petrolera, nuclear, del papel y alimentos, entre otras, (Videla, 1996). Se ha estimado que el 20 o 30\% de las fallas en tuberías de transporte de hidrocarburos, están relacionadas a problemas de corrosión microbiológica, afectando la parte interna y externa de éstas. En EUA, se gastan del orden de 120 millones de dólares al año en biocidas para combatir este tipo de corrosión. Estos tratamientos no son solamente costosos, sino que pueden tener un efecto dañino al medio ambiente y a la vida humana (Thompson, 2000).

Los microorganismos pueden generar productos metabólicos de posible actividad corrosiva, afectando el medio circundante a la interfase metal/solución, este efecto se magnifica por la acción sinérgica de la asociación de microorganismos presentes en el medio (consorcios microbianos). El metabolismo y velocidad de crecimiento de éstos, pueden explicar por qué ciertas interfaces metal/solución pueden incrementar significativamente las velocidades de corrosión que experimentan (Treseder, 1991). Los procesos metabólicos de los microorganismos son sustentados por reacciones químicas para la generación de energía, a través de la asimilación de nutrientes que se encuentran en el medio circundante. Según Videla dichos procesos influyen el comportamiento de la corrosión de los materiales metálicos, introduciendo o incrementando la heterogeneidad de la superficie metálica a través de la:

- Destrucción de películas protectoras de las superficies metálicas.

- Generación de ambientes ácidos localizados.
- Creación de depósitos corrosivos.

- Alteración de reacciones anódicas y catódicas.

- Interferencia en mecanismos de protección contra la corrosión.

Se debe tomar en cuenta que la simple presencia de microorganismos en un sistema, no necesariamente indica que causen problemas de corrosión, ya que poblaciones bacterianas que son problemáticas en un sistema específico, pueden ser inertes en otro sistema diferente (NACE, 1994). La tabla 1 presenta los grupos de microorganismos que están comúnmente relacionados con los procesos de corrosión microbiológica.

Tabla 1. Microorganismos comúnmente relacionados a la corrosión microbiológica (Treseder, 1991; Martín, 1999)

\begin{tabular}{cccc}
\hline Género o especie & $\mathrm{pH}$ & Temp. $\left({ }^{\circ} \mathrm{C}\right)$ & $\begin{array}{c}\text { Requerimiento de } \\
\text { oxígeno }\end{array}$ \\
\hline $\begin{array}{c}\text { Desulfovibrio } \\
\text { desulfuricans }\end{array}$ & $4-8$ & $10-40$ & Anaerobia \\
Desulfotomaculum & $6-8$ & $10-40$ & Anaerobia \\
Nigrificans & & $45-75$ & Anaerobia \\
(Clostridium) & $\ldots$ & $10-40$ & Anaerobia \\
Desulfomonas & $0.5-8$ & $10-40$ & Aerobia \\
Thiobacillus & $1-7$ & $10-40$ & Aerobia \\
thiooxidans & & & \\
Thiobacillus & $7-10$ & $20-40$ & Aerobia \\
ferroxidans & & & \\
Gallionella & $7-10$ & $20-40$ & Aerobia \\
Sphaerotillus & $4-9$ & $20-40$ & Aerobia \\
Pseudomonas & $4-8$ & $20-40$ & Aerobia \\
P. Aeruginosa & $3-7$ & $10-45$ & $\ldots$ \\
Cladosporium & & & \\
resinae & & & \\
\hline
\end{tabular}


El factor clave para la alteración de las condiciones de la superficie metálica y con esto, un incremento (o retardo) de la velocidad de corrosión es el biofilm (Edyvean et al., 1991).

Un biofilm o biopelicula es un conglomerado microbiano compuesto por bacterias, algas y otros microorganismos que está constituido de una matriz gelatinosa de material polimérico extracelular (MPE) con un elevado contenido de agua (aproximadamente del 80 al 95\% de la masa) y células microbianas (Heitz, 1996). Esta película biológica modifica las condiciones de la interfase metal/solución, donde las reacciones que se producen entre los metabolitos microbianos y la superficie metálica tienen lugar por debajo o por dentro de la estructura del biofilm.

Se denomina bioprotección a la relación entre los múltiples mecanismos, metal / biota / electrolito, que ocasionan un efecto sinérgico en la inhibición de la corrosión (Videla, 1996). La inhibición originada por los microorganismos se debe a la acción neutralizadora de las sustancias corrosivas presentes en el ambiente, esto a través de la modificación en la concentración de oxígeno y del pH del medio (Flemming, 2002), formando películas protectoras que impiden las reacciones entre el medio corrosivo y la superficie metálica (Rajasekar, 2005), esto último a través de estabilizar la película de óxido, la cual dificulta la difusión de sustancias corrosivas en la superficie del metal o también disminuyendo la corrosividad de medio.

Este estudio tiene el objetivo de emplear las técnicas electroquímicas potencial a circuito abierto, resistencia a la polarización lineal, y espectroscopia de impedancia electroquímica para el estudio del fenómeno de la corrosión microbiológica, empleando acero de bajo carbono SAE 1018 sometido a la acción de diferentes cargas microbianas, a fin de poder correlacionar los diferentes parámetros estudiados.

Entre las técnicas electroquímicas que se han empleado para el estudio de la corrosión microbiológica se encuentran (Cottis et al.,1999; Masfeld, 1978):

- Potencial a circuito abierto (OCP),

- Resistencia a la polarización lineal (LPR) y

- Espectroscopia de impedancia electroquímica (EIS).

\section{Potencial a circuito abierto}

Se determina midiendo la diferencia de potencial entre el metal sumergido en un medio iónico y un electrodo de referencia (generalmente un electrodo de calomel saturado SCE). La magnitud y signo de este parámetro depende del metal, de la composición del medio, temperatura, de las condiciones hidrodinámicas, $\mathrm{pH}$, etc. En la práctica resulta ser una prueba muy sencilla de realizar, su desventaja radica en que no determina valores y cambios en velocidades de corrosión.

\section{Resistencia a la polarización lineal}

Ha sido aplicada a estudios de corrosión microbiológica durante muchos años, tanto en el campo como en el laboratorio (Videla, 1996). Esta técnica está basada en la aplicación de pequeños potenciales y el registro de la respuesta de corriente. Asumiendo una relación lineal entre estas dos variables se calcula una resistencia llamada resistencia a la polarización que se interpreta como el comportamiento resistivo de la interfase metal/ solución, que para un control activacional coincide con la Resistencia a la transferencia de carga (Rtc) de la doble capa electroquímica. La linealidad entre cambios de potencial y corriente, es relacionada por la ecuación de Stern \& Geary donde la corriente de corrosión es inversamente proporcional a la resistencia a la polarización:

$$
\begin{aligned}
& i_{\text {corr }}=\frac{B}{R_{p}} \\
& B=\frac{b_{a} b_{c}}{2.303\left(b_{a}+b_{c}\right)}
\end{aligned}
$$

donde:

$b_{a}:$ pendiente de Tafel anódica.

$b_{c}$ : pendiente de Tafel catódica.

$R_{\mathrm{p}}$ : resistencia a la polarización.

$\mathrm{i}_{\text {corr }}$ : densidad de corriente de corrosión.

Esta técnica se aplica en casos donde la corrosión es uniforme; se caracteriza por su rapidez y simplicidad, permite calcular velocidades de corrosión instantáneas. No es recomendada para ataque localizado ni en casos en que el control sea por transferencia de masa.

Debido a las pequeñas polarizaciones aplicadas, se puede monitorear la Rp sin alterar la superficie ni la presencia biotica del medio, razón por la que la técnica como extrapolación de Tafel, en la que se aplican grandes polarizaciones no es recomendada en el estudio de la corrosión microbiológica. 
DOI: http://dx.doi.org/10.22201/fi.25940732e.2009.10n1.002

Corrosión microbiológica en aceros de bajo carbono

\section{Espectroscopia de impedancia electroquímica}

Consiste en la aplicación de un voltaje alterno fijo a un electrodo variando la frecuencia, obteniendo la respuesta en corriente y calculando la impedancia a su respectiva frecuencia. Debido a los bajos potenciales aplicados se considera una técnica de baja perturbación al sistema y permite discriminar y medir componentes resistivos, capacitivos e inductivos de una interfase; entre ellos la Rtc, pudiendo modelar el comportamiento electroquímico por medio de circuitos eléctricos equivalentes. Esta técnica determina si existe un control por transferencia de masa, además de ser útil en los estudios de películas no conductoras o poco conductoras que recubren metales como las películas orgánicas. La desventaja en estudios de corrosión microbiológica es que los circuitos eléctricos equivalentes son difíciles de modelar, como el caso de interfases complejas que involucran el desarrollo de una biopelícula discontinua y de espesor variable en toda la superficie.

\section{Materiales y métodos}

Las cargas microbianas estudiadas fueron un consorcio microbiano procedente de una muestra tomada en la zona de Chinampas de Xochimilco, México D.F., las restantes son bacterias aisladas del mencionado consorcio, cuya identificación está en proceso, por lo que se les referirá a ellas como bacteria $25 \mathrm{sp}$. y bacteria $33 \mathrm{sp}$.

Debido al amplio uso del acero de bajo carbono SAE 1018, se realizaron estudios electroquímicos empleando probetas de este material (WE) con área de exposición de $1.5 \times 1.5 \mathrm{~cm}$, un acabado superficial a espejo para evitar el estancamiento de nutrientes y así posibles zonas de crecimiento localizado de los microorganismos. Se empleó un electrodo saturado de calomel como electrodo de referencia (ESC) y una barra de grafito de alta pureza como electrodo auxiliar (AE).

Los técnicas electroquímicas utilizadas fueron potencial a circuito abierto (OCP), resistencia a la polarización (Rp) y espectroscopia de impedancia electroquímica (EIS), para la cual se utilizó un potencióstato marca ACM instruments modelo Gill AC con interfase electrónica a una computadora personal, con registro y procesamiento de datos por medio de ACM software.

Los electrodos fueron sumergidos en el medio de cultivo NACE a $37^{\circ} \mathrm{C}$ (tabla 2) previamente esterilizado por medio de autoclave ( 20 minutos, $\left.121^{\circ} \mathrm{C}, 15 \mathrm{psi}\right)$. La composición del medio se presenta en la tabla 2. Los electrodos y termómetro se esterilizaron en frío. Los ensayos electroquímicos fueron realizados con presencia (biótico) y ausencia (abiótico) de microorganismos, designando una celda de trabajo y una de control, respectivamente. Para el caso biótico se realizaron conteos microbianos para la construcción de la curva de crecimiento microbiano.

Tabla 2. Composición del medio de cultivo NACE

\begin{tabular}{cc}
\hline Compuesto & Cantidad \\
\hline Citrato Férrico & $0.10 \mathrm{gr}$ \\
Estracto de levadura & $3 \mathrm{gr}$ \\
Peptona & $5 \mathrm{gr}$ \\
$\mathrm{MgCl}_{2}$ & $8.8 \mathrm{gr}$ \\
$\mathrm{Na}_{2} \mathrm{SO}_{4}$ & $3.3 \mathrm{gr}$ \\
$\mathrm{CaCl}_{2}$ & $1.8 \mathrm{gr}$ \\
$\mathrm{KCl}$ & $0.6 \mathrm{gr}$ \\
$\mathrm{NaCl}$ & $19.5 \mathrm{gr}$ \\
$\mathrm{NaHCO}$ & $0.16 \mathrm{gr}$ \\
Agua destilada & $1000 \mathrm{ml}$ \\
\hline
\end{tabular}

Con lo que respecta al tiempo, se estudió el comportamiento electroquímico para periodos de 48 horas y 28 días. Para el primer caso, se hicieron mediciones aproximadamente cada hora.

En el segundo caso las mediciones fueron hechas los días 1,2 ,4, 7, 10,15, 22 y 28. Se construyeron las curvas de crecimiento microbiano para las diferentes bacterias estudiadas y tiempos de prueba, empleando la técnica de recuento en placa (Ingraham et al., 2000).

La secuencia de aplicación de las técnicas electroquímicas fue la siguiente: se esperó la estabilización del OCP mediante su monitoreo durante $55 \mathrm{~min}$. Inmediatamente se efectuó el ensayo de EIS aplicando un barrido de frecuencias de $10^{5} \mathrm{~Hz}$ a $10^{-2} \mathrm{~Hz}$, con una amplitud del voltaje de $10 \mathrm{mV}$. Debido a la baja perturbación en el sistema electroquímico que ocasiona la técnica EIS, se esperó 10 minutos para la estabilización del OCP y así realizar el ensayo de Rp aplicando una velocidad de barrido de $10 \mathrm{mV} / \mathrm{min}$, con una polarización de $20 \mathrm{mV}$ catódicos a $20 \mathrm{mV}$ anódicos con respecto al potencial de corrosión (Ecorr) obtenido en la estabilización. Los resultados obtenidos de impedancia, potencial y densidad de corriente se analizaron mediante MATHCAD 12 para ajustar los semicírculos y rectas resultantes usando regresión por mínimos cuadrados y así poder determinar el valor de la Rtc. 
Después de cada ensayo, las muestras fueron almacenadas en un desecador para posteriormente observar la superficie de éstas en el microscopio electrónico de barrido (MEB).

\section{Resultados}

En las figuras 1 y 2 se presentan las curvas de crecimiento para el consorcio microbiano y bacterias 33 sp. y 25 sp., durante un periodo de exposición de 28 días y 48

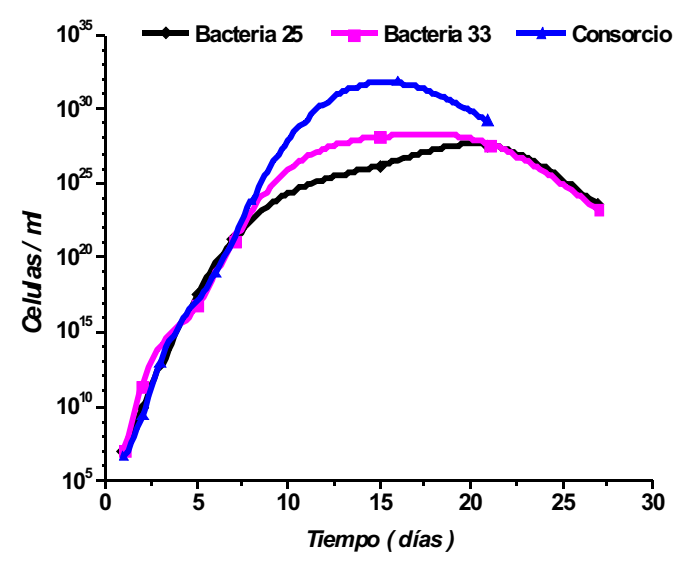

Figura 1. Curva de crecimiento microbiano durante 28 dias de exposición, se aprecia la fase exponencial, estacionaria $y$ de muerte celular

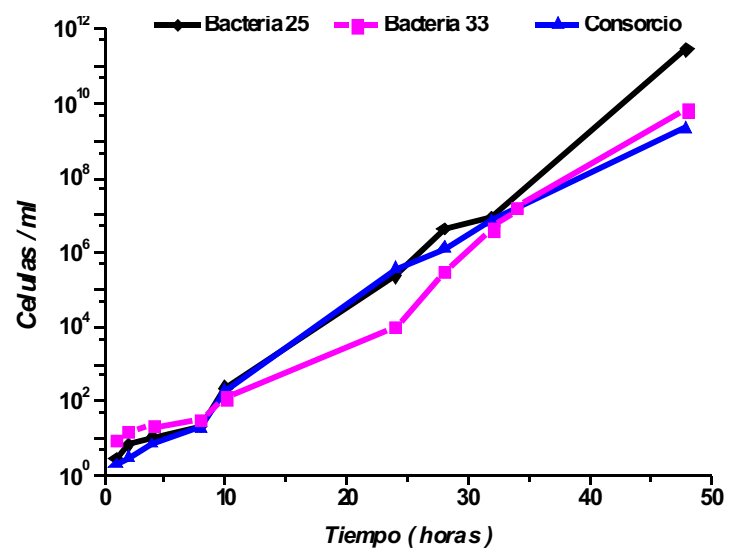

Figura 2. Curva de crecimiento microbiano durante 48 horas de exposición, se aprecia la fase de retardo horas, respectivamente. En ellas se observan las fases de crecimiento exponencial, estacionaria y de muerte celular, concordando con lo citado en la literatura (Ingraham et al., 2000).

Los valores de OCP obtenidos en las pruebas de 28 días y 48 horas se presentan en las figuras 3 y 4 , respectivamente, para las pruebas de trabajo y de control.

Los valores de Rtc obtenidos en las pruebas de 28 días y 48 horas se presentan en las figuras 5 y 6 respectivamente, para las diferentes pruebas.

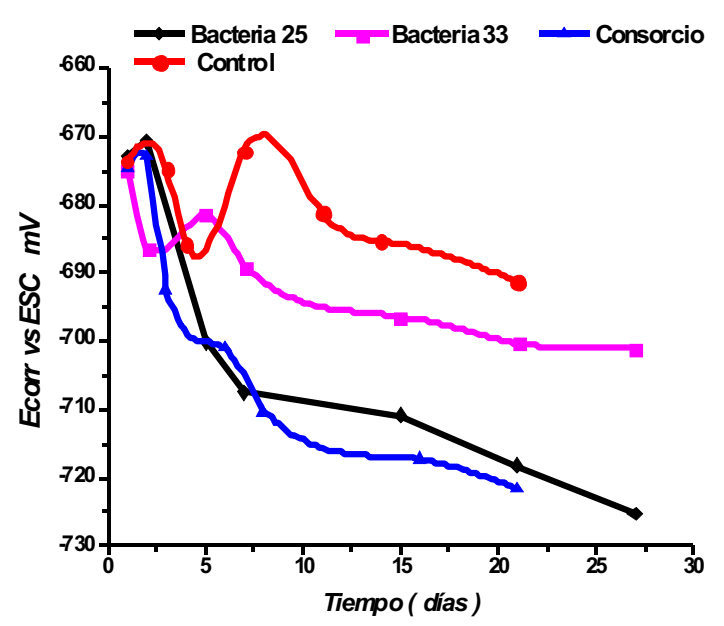

Figura 3. Valores de OCP para las pruebas de trabajo $y$ control en un periodo de 28 dias

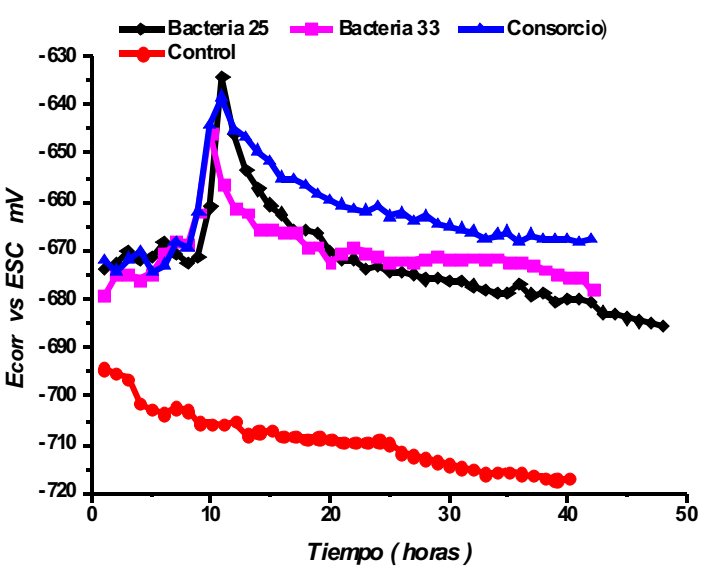

Figura 4. Valores de OCP para las pruebas de trabajo $y$ control en un periodo de 48 horas 


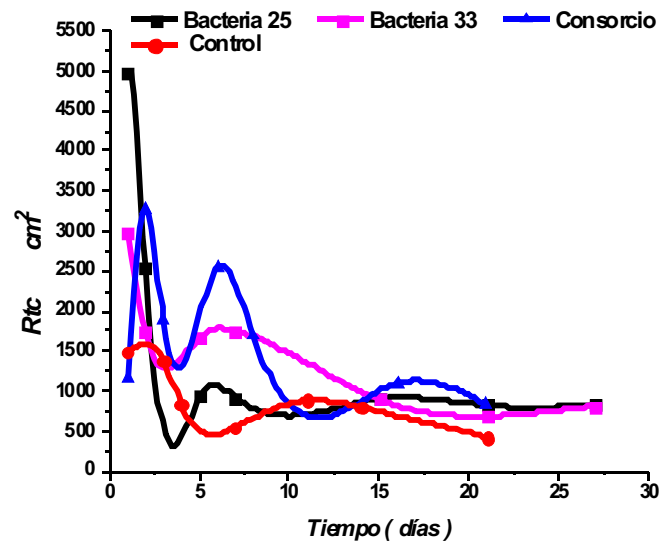

Figura 5. Valores de Rtc para las pruebas de trabajo y control en un periodo de 28 dias

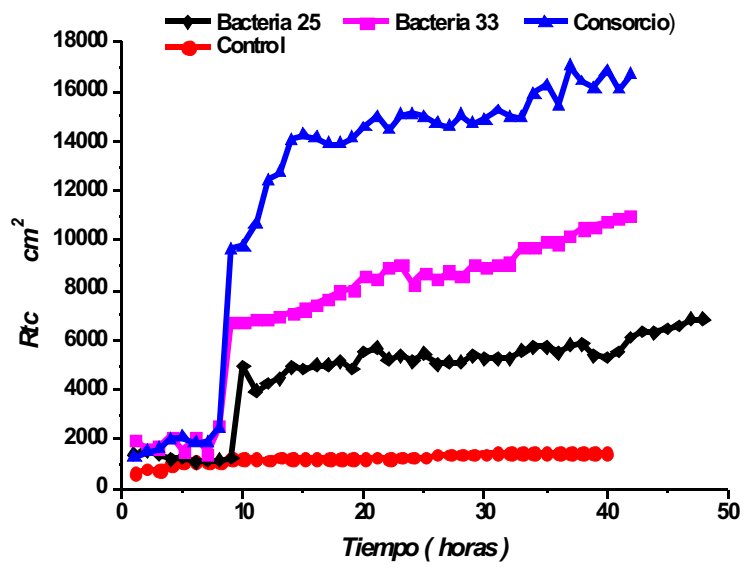

Figura 6. Valores de Rtc para las pruebas de trabajo y control en un periodo de 48 horas

\section{Discusión}

En los conteos para las pruebas de 48 horas, el cambio de fase de retardo a exponencial se presenta aproximadamente entre 9 a 10 horas para los tres sistemas. La fase estacionaria se alcanza entre los días 13 y 15 durante la prueba de 30 días, con un mayor número de células en el consorcio microbiano, seguido por las bacterias $33 \mathrm{sp}$. y $25 \mathrm{sp}$.

El consorcio microbiano y bacteria 33 sp. alcanzan la fase de muerte celular alrededor de los 19 días, mientras que para la bacteria $25 \mathrm{sp}$. ésta se presenta alrededor del día 22.

Esta última fase se presentó en el consorcio microbiano en un menor tiempo, debido a la disminución de nutrientes en el medio ocasionado por la mayor cantidad de células en la fase estacionaria con respecto a los demás sistemas bióticos.

De igual forma, debido al menor número de células, la bacteria $25 \mathrm{sp}$ presenta la fase de muerte en un tiempo más prolongado con relación a los demás sistemas.

\section{OCP 48 horas}

Los comportamientos de OCP obtenidos en los tres sistemas bióticos experimentan un cambio abrupto aproximadamente entre 8 y 10 horas de exposición, aumentando hasta un valor máximo y disminuyendo con rapidez hasta alcanzar una velocidad de cambio constante. La prueba de control presenta una tenue disminución de OCP sin experimentar cambios abruptos.

\section{OCP 28 días}

Los resultados indican que durante los primeros 7 días de exposición el consorcio microbiano y bacteria $25 \mathrm{sp}$ tienen comportamientos similares y diferentes a los sistemas restantes. Después de este día, la tendencia de todos los sistemas es a disminuir con prácticamente una misma velocidad de cambio.

Los mayores valores de OCP después del día 7 fueron los registrados por la prueba de control, y los menores los del consorcio microbiano.

\section{Rtc 48 horas}

$\mathrm{Al}$ igual que los resultados de OCP, los valores de Rtc para los sistemas bióticos presentan cambios abrutos alrededor de 9 a 10 horas de exposición, hasta alcanzar un valor en el que se presenta una velocidad de aumento uniforme para todos los sistemas; las mayores velocidades de corrosión son las ocasionadas por la prueba de control, seguida por la bacteria $25 \mathrm{sp}$, bacteria $33 \mathrm{sp}$ y por último las del consorcio microbiano, lo que indica que el efecto de éste último en las velocidades de corrosión es menor en cualquier momento, durante el periodo de tiempo estudiado con respecto a los demás sistemas; en contraparte, la prueba de control resultó con las mayores velocidades de corrosión con valores prácticamente constantes.

\section{Rtc 28 días}

Durante los primeros siete días de exposición los comportamientos de cada uno de los sistemas es diferente, presentando aumentos y disminuciones en diferentes instantes. Posterior a este día todos los sistemas tienden 
a disminuir gradualmente hasta que a partir del día 15 tienden prácticamente el mismo valor de Rtc. La prueba de control registró las mayores velocidades de corrosión a partir del día 3; en contraparte, las menores velocidades de corrosión las registró el consorcio microbiano durante el tiempo en estudio, esto ratifica los resultados obtenidos en la prueba de 48 horas.

Para comparar los resultados de Rtc obtenidos por LPR y EIS, se eligió aleatoriamente un par de datos obtenidos por ambas técnicas; esto se presenta en las figuras 7 a y 7 b para la prueba de control a 4 y 28 horas de exposición.

Se observa que las tendencias se conservan con pequeñas diferencias numéricas, lo que se considera como una buena similitud entre ambas.

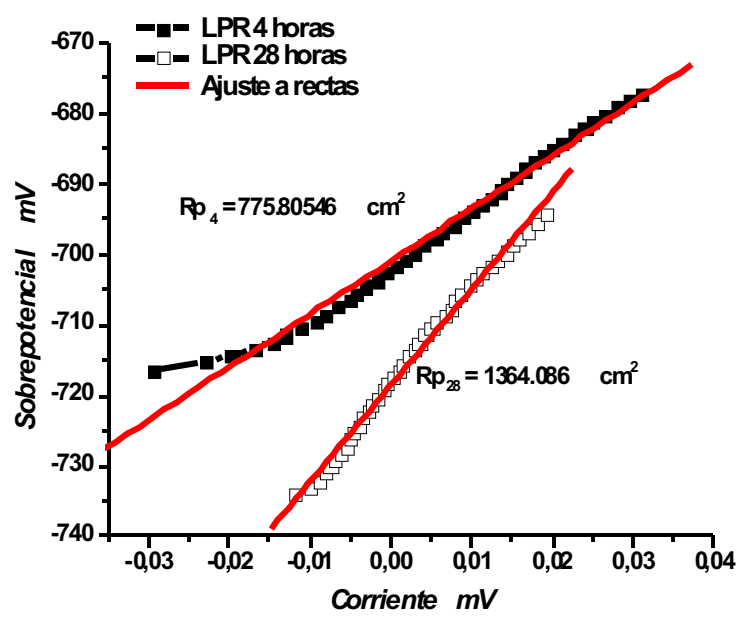

Figura 7a. Ajuste de datos para obtener la Rp

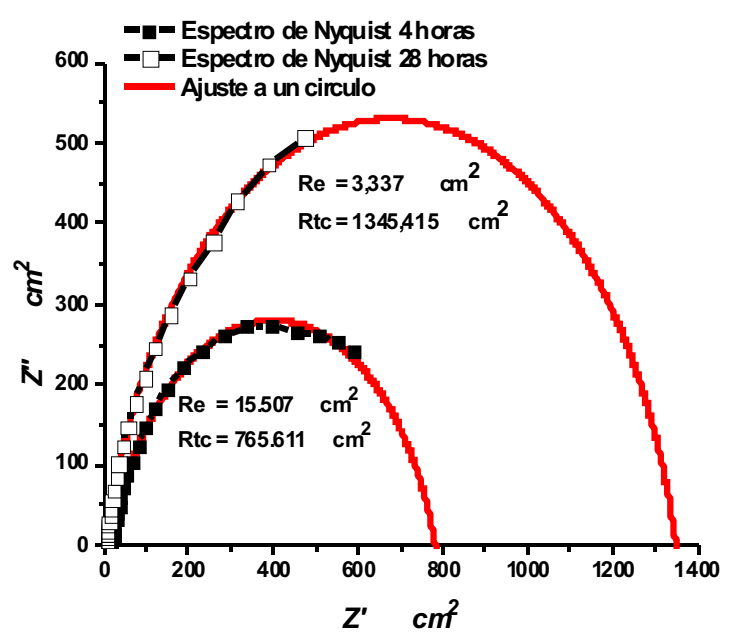

Figura 76 . Ajuste a un círculo de los resultados
En la figura 8 se muestran los espectros de Nyquist del día 1 y 28 de exposición para las diversas pruebas de trabajo y control.

En ellas se aprecia que en las frecuencias estudiadas el control activacional prevalece desde el inicio hasta el final de cada una de las pruebas.

Se nota que el crecimiento microbiano induce un aumento en las velocidades de corrosión, menores a las obtenidas en el sistema abiotico. Al permanecer constante el crecimiento en la fase estacionaria, la velocidad de variación de Vcorr disminuye. La disminución o aumento del OCP indica una mayor o menor tendencia del metal a corroerse, esto no debe de interpretar que el proceso ocurre a mayores o menores velocidades de corrosión, por lo que es preciso presentar las relaciones existentes entre estos dos parámetros.

La explicación de los cambios abruptos de Rtc en las pruebas de 48 horas en los sistemas bióticos se expone en la figura 9, donde el cambio abrupto de este parámetro ocurre simultáneamente con el cambio de fase de retardo a exponencial en la curva de crecimiento. Los cambios abruptos de OCP y Rtc en los sistemas bióticos, suceden en el mismo instante, lo que indica que ambos son afectados por el cambio de fase en la curva de crecimiento.

En la figura 9, después de 10 horas de exposición una disminución en las velocidades de corrosión se relaciona con una disminución en el OCP. Para apoyar una posible explicación de esta relación se presentan en la tabla 3 las mediciones de $\mathrm{pH}$ al inicio y final de cada una de las pruebas de 48 horas.

Debido a que los valores de $\mathrm{pH}$ están dentro de la zona neutra, la reacción catódica queda controlada por la reducción del oxígeno. En el diagrama de Evans - Tafel de la figura 10, se plantea una disminución del oxígeno debido a la respiración microbiana, lo que ocasiona una polarización catódica, esto es un cambio de pendiente en la recta catódica que induce una disminución simultánea en los valores de OCP y Vcorr. Se considera que una disminución del potencial de corrosión, no necesariamente induce un aumento en las velocidades de corrosión.

Los resultados de los ensayos electroquímicos para las pruebas de 30 días son respaldados por las observaciones hechas en el MEB en las probetas del día final para cada uno de los sistemas estudiados, que se muestran en las figuras 11-14. Al comparar visualmente las imágenes es notable que el medio de cultivo ocasionó mayores daños superficiales al material, provocando ataques localizados en menor densidad, pero mayores en tamaño. 


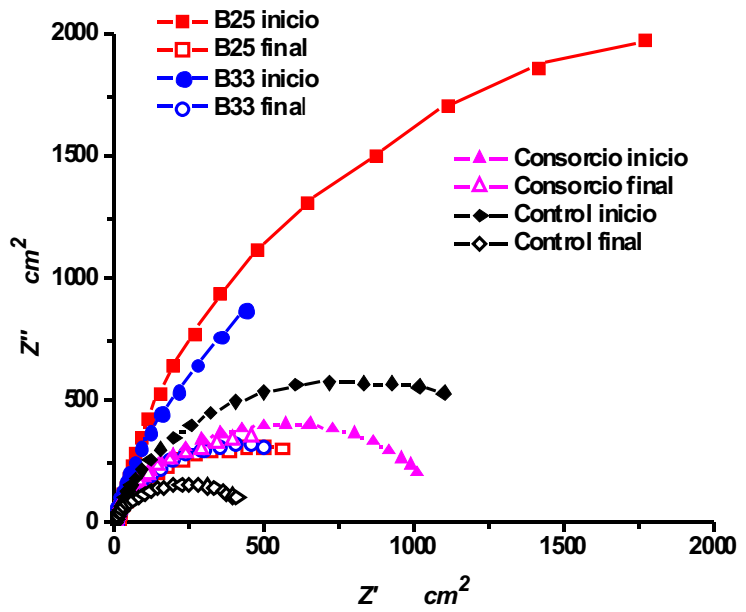

Figura 8. Espectros de Nyquist con barridos de frecuencia de $10^{5}$ hasta $10^{-2} \mathrm{~Hz}$

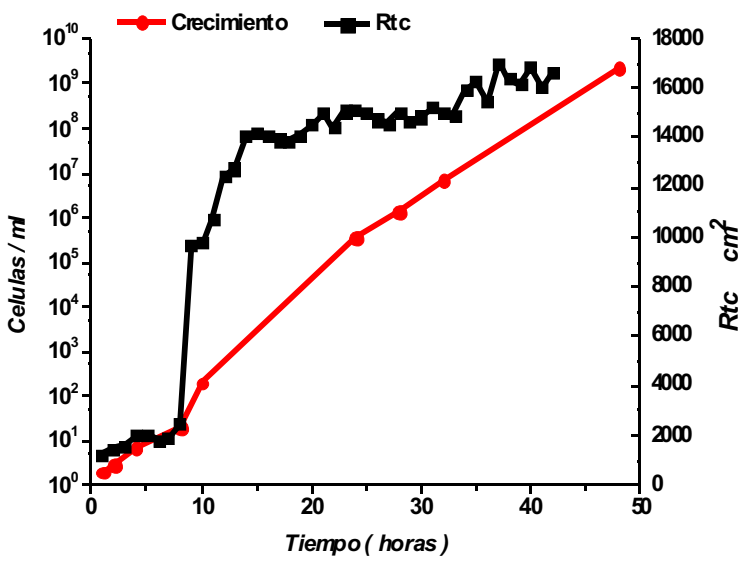

Figura 9. Efecto del cambio de fase de retardo a exponencial en los valores de (Rtc)

Tabla 3. Valores del pH antes y después de las pruebas de 48 horas

\begin{tabular}{ccc}
\hline Microorganismo & $\mathrm{pH}$ inicial & $\mathrm{pH}$ final (48 horas) \\
\hline Consorcio & 7.35 & 7.54 \\
Bacteria 25 sp. & 6.72 & 6.94 \\
Bacteria 33 sp. & 7.14 & 7.28 \\
\hline
\end{tabular}

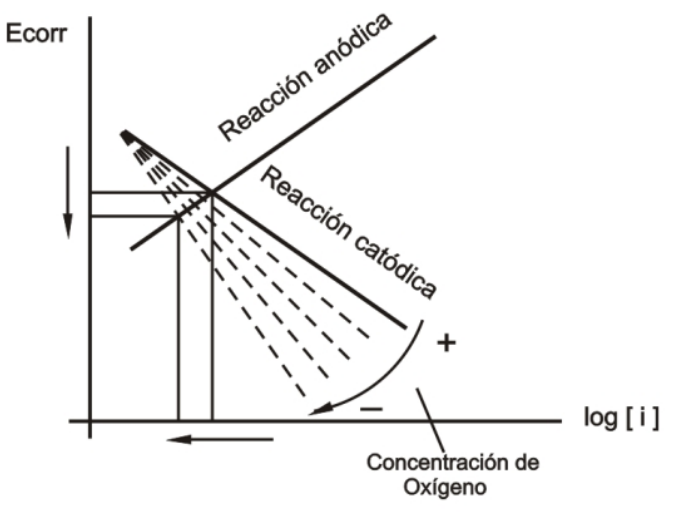

Figura 10. Mecanismo de polarización catódica provocado por una disminución en la concentración de oxígeno

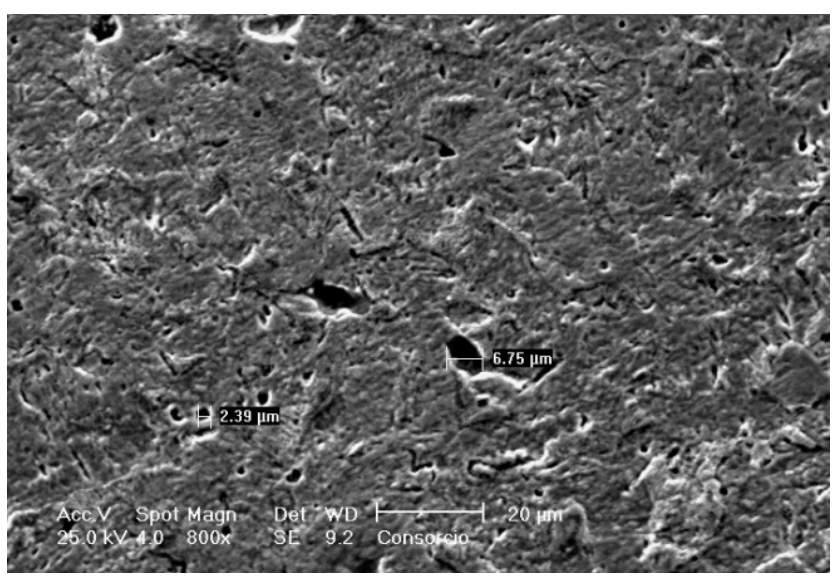

Figura 11. Superficie expuesta al consorcio microbiano, $800 x$

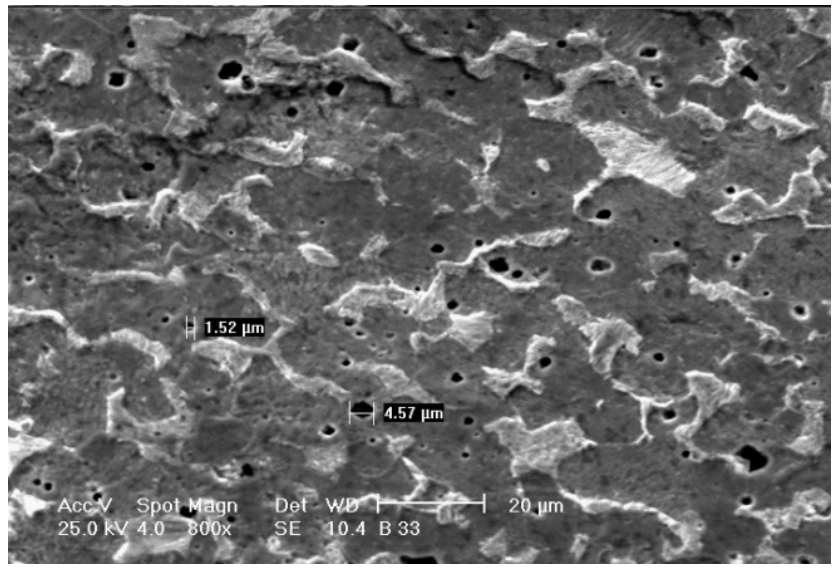

Figura 12. Superficie expuesta a la bacteria 33 sp., 800x 


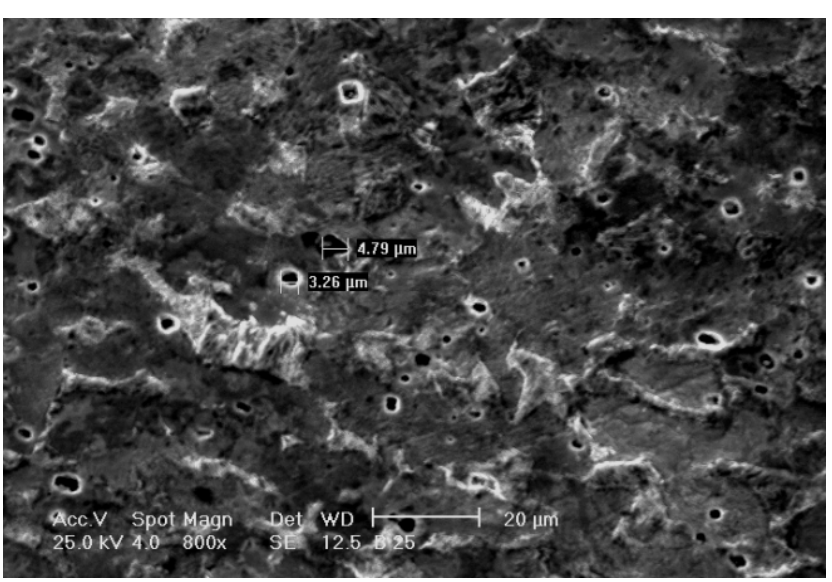

Figura 13. Superficie expuesta a la bacteria 25 sp., $800 x$

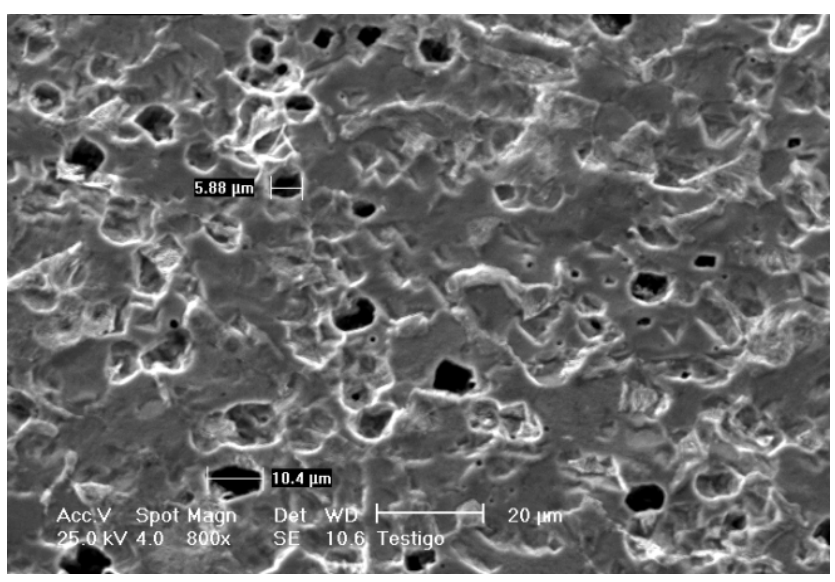

Figura 14. Superficie expuesta únicamente al medio de cultivo, se observan menores ataques localizados, $800 x$

A pesar de no haber realizado ensayos que proporcionen más información mecanística para dar una explicación certera del comportamiento de los sistemas estudiados en la prueba de 30 días se propone el siguiente planteamiento:

Los daños localizados en las superficies expuestas al medio abiótico fue debido a la formación de una capa pasiva en la superficie en los primeros días de exposición, que conforme aumentó el tiempo de exposición, aunado con la alta concentración de cloruros en el medio de cultivo ésta se rompió, provocando la formación y crecimiento de picaduras. Estos daños fueron inhibidos en los sistemas con presencia de bacterias debido a cualquiera de las siguientes posibilidades:

- La presencia de un biofilm protector aisló gradualmente la superficie del acero con el medio de cultivo conforme esta capa aumentaba en espesor; sin embargo, las picaduras observadas en el MEB en los sistemas bióticos fue causada por la generación de celdas de concentración provocadas por la presencia del biofilm.

- Se considera que las velocidades de corrosión fueron menores en los medios bióticos debido a la continua disminución de oxigeno ocasionada por el aumento en número de células, que también dio lugar a la formación de un biofilm que ocasionó la formación y crecimiento gradual de celdas de concentración de aireación diferencial, logrando simultáneamente un efecto de inhibición por parte de la respiración microbiana y un efecto corrosivo por parte de la presencia del biofilm. Ambas jugaron un papel de competencia en las velocidades de corrosión, con un mayor efecto de inhibición antes del día 15, después el crecimiento microbiano deja de influir y se acentúa el efecto corrosivo debido al biofilm. Las picaduras observadas en el medio biótico son debidas a las celdas de concentración generadas por el biofilm, siendo menor el efecto de éste en número y tamaño de picaduras que en condiciones abioticas.

\section{Conclusiones}

- Los microorganismos estudiados completan en 22 días las cuatro fases características en una curva de crecimiento.

- El cambio de fase de retardo a exponencial en la curva de crecimiento afecta abruptamente el potencial y velocidad de corrosión en todos los sistemas bióticos estudiados.

- La disminución de la velocidad y potencial de corrosión que experimentan los sistemas bióticos durante las pruebas de 48 horas, se explica por medio de una polarización catódica que induce una disminución simultánea en estos valores.

- Durante los primeros 15 días de exposición, las mayores velocidades de corrosión fueron las registradas por el medio sin bacterias, lo que indica un mayor efecto corrosivo del medio de cultivo y la acción protectora por parte de los microorganismos estudiados.

- El efecto corrosivo de las bacterias 25 sp y $33 \mathrm{sp}$ es mayor que la del consorcio microbiano durante los primeros 15 días de exposición.

- Las técnicas electroquímicas de LPR y EIS presentaron una buena correlación en los resultados de Rtc, que enfatiza la efectividad de éstas, lo cual 
DOI: http://dx.doi.org/10.22201/fi.25940732e.2009.10n1.002

Corrosión microbiológica en aceros de bajo carbono

muestra la conservación de tendencias, así como mínimas diferencias numéricas entre ellas. Lo anterior se verifica con base en los resultados obtenidos a través del ajuste por mínimos cuadrados, método que tiene la ventaja de eliminar cualquier desviación producto del empleo de métodos gráficos, que es la causa principal de los errores que se presentan en algunos de los trabajos publicados. Como prueba de la confiabilidad del método se puede referir el lector a las gráficas de las figuras 7 a y $7 \mathrm{~b}$ en las cuales los valores de resistencia a la polarización se corresponden a los de resistencia a la trasferencia de carga, dado que se considera que la resistencia del electrolito es mucho menor, por lo que los valores de resistencia a la polarización y a la trasferencia de carga son prácticamente iguales.

\section{Recomendaciones}

- Debido a que este trabajo de investigación es una primera aproximación al estudio de la corrosión microbiológica, no fue posible incluir técnicas de uso más reciente como Ruido electroquímico; sin embargo, en estudios posteriores se pretende emplear ésta para compararla con las ya utilizadas y elegir la más apropiada para el estudio de este tipo de corrosión, con base a economía, efectividad, rapidez y simplicidad.

- El barrido de frecuencias de $10^{5}$ hasta $10^{-2}$, no fue suficiente para descartar la posibilidad de un control difusional o aportar información adicional para determinar un mecanismo de ataque, por lo que se recomienda emplear frecuencias menores de 1 hasta 2 décadas.

\section{Agradecimientos}

Los autores agradecen el apoyo económico otorgado para la realización del presente proyecto por DGAPA IN102706-2. Así mismo se le agradece al Biol. Germán Álvarez Lozano y al Mtro. Carlos Rodríguez por el apoyo técnico brindado en la parte microbiológica y en la obtención de imágenes en el microscopio electrónico de barrido.

\section{Referencias}

Borestein S.W. Microbiologically Influenced Corrosion. Handbook. New York. 1994.

Cottis R., Turgoose S. Electrochemical Impedance and Noise. Serie Corrosion Testing Made Easy. USA. NACE International.1999.

Cottis R.A., Turgoose S. Electrochemical Kinetics and Thermodynamics. USA. NACE International. 1998.

Edyvean R.G., Videla H.A. Biological Corrosion. USA. Interdisciplinary Science Reviews. 1991. Pp.16, 267.

Flemming C. Biofouling in Water Systems (cases) Causes and Countermeasures. Montana, USA. Appl Microbiol Biotechnol. 2002.

Heitz H.E., Flemming C., Sand W. Microbially Influenced Corrosión of Materials. Montana, USA. Springer 1996.

Ingraham J.L., Ingraham C.A. Introducción a la microbiología. Vol.1 y 2. Barcelona. Ed. Reverte S.A. 2000.

Masfeld F. Polarization Resistence Measurement-Experimental Procedure and Evaluation of Test Data, in Electrochemical Techniques for Corrosion. Houston. R. Robain Editor. NACE. 1978.

Merck E., Manual de medios de cultivo. Darmstandt, Alemania. Ed. Merck. 1994.

NACE Field Monitoring of Bacterial Growth in Oilfield Systems. Sytandard TMO194-94. 1994.

Pope D.H. A Study of Microbiologically Influenced Corrosion and a Practical Guide for its Treatment and Prevention. Final report, EPRI CS-5495. Electric Power Research Institute. 1986.

Rajasekar A., Maruthamuthu S., Muthukumar N., Mohanan S., Subramanian P., Palaniswamy N. Bacterial Degradation of Naphtha and its Influence on Corrosion. Corrosion Science 47. 2005.

San Martín R.G. Microbilogical Techniques to Evaluate Biocides in Secondary Recovery Systems. UMIST, UK. MsC. Disertation. 1999.

Treseder R.S. NACE Corrosion Engineers Reference Book. Second edition, Houston. NACE International.1991.

Videla H.A. Manual of Biocorrosión. Florida USA. Lewis Publishers. 1996.

Neil. D., Thompson G. Estudio económico en líneas de transmisión. Tesis (Doctorado) Ohio. 2000. Disponible en: http://www.corrosioncost.com/pdf/gasliquid.pdf 
DOI: http://dx.doi.org/10.22201/fi.25940732e.2009.10n1.002

O. Medina-Custodio, A. Ortiz-Prado, V.H. Jacobo-Armendáriz y R. Schouwenaars-Franssens

\section{Semblanza de los autores}

Oscar Medina-Custodio. Es ingeniero mecánico electricista graduado por la Universidad Juárez Autónoma de Tabasco en el año 2002. En 2006, obtuvo el grado de maestro en ingeniería con mención honorífica, en la orientación de mecánica aplicada en el programa de posgrado en ingeniería de la Universidad Nacional Autónoma de México. Está orientado en el campo de la mecánica teórica y experimental, además de tener conocimientos en la ingeniería de la corrosión. Ha publicado 3 artículos como autor principal.

Armando Ortiz-Prado. Se graduó como ingeniero mecánico electricista por la Facultad de Ingeniería de la UNAM en 1980, posteriormente obtuvo la maestría y el doctorado en ingeniería mecánica. Es profesor desde hace 28 años impartiendo cátedra principalmente en las áreas de procesos de manufactura y ciencia de materiales. Es profesor titular "C" de tiempo completo definitivo y tutor doctoral de los posgrados de ciencia e ingeniería de materiales y de ingeniería. Sus líneas de investigación son modelado de procesos de conformado mecánico, análisis de falla, comportamiento mecánico de biomateriales y corrosión en equipo electrónico. Ha desarrollado proyectos y estudios para diferentes empresas privadas y entidades gubernamentales, orientados al desarrollo y mejora de métodos de manufactura, desarrollo de materiales, corrosión y análisis de falla; lo cual se traduce en más de 50 estudios y asesorías para la industria, 12 proyectos de investigación y desarrollo tecnológico. A la fecha, suma más de 90 tesis de licenciatura concluidas, así como 12 de maestría y una de doctorado. Su experiencia se ha transmitido a través de 7 textos para licenciatura en las áreas de manufactura, materiales y corrosión. Asimismo, su productividad incluye más de 15 artículos en congresos internacionales, más de 80 artículos en congresos nacionales y más de 15 artículos en revistas arbitradas. Fue galardonado por la UNAM con la Distinción Universidad Nacional para Jóvenes Académicos (Docencia en ciencias exactas) en 1989. Su productividad académica y científica ha sido reconocida por la UNAM, a través del otorgamiento del nivel D del PRIDE y por el CONACYT con el nombramiento de Investigador Nacional Nivel I.

Víctor Hugo Jacobo-Armendáriz. Ingeniero mecánico electricista (Ingeniería industrial) por la Facultad de Ingeniería de la UNAM, en donde también obtuvo los grados de maestro y doctor en ingeniería. Actualmente funge como profesor titular "B" de tiempo completo definitivo en el área de manufactura y materiales, en la propia FI-UNAM. Su línea se ha orientado al análisis de falla, ciencia de materiales y sistemas expertos. De 13 años a la fecha, ha participado en diversos proyectos y estudios al desarrollo y mejora de métodos de manufactura, corrosión y análisis de falla. Ha dirigido 10 tesis de licenciatura y publicado 2 textos (análisis de falla, corrosión y protección), más de 5 artículos en congresos internacionales, más de 25 en congresos nacionales y más de 10 en revistas arbitradas. Actualmente es investigador nacional nivel 1 del SNI.

Rafael Schouwenaars-Franssens. Obtuvo el grado de maestro en ingeniería de minas por la Universidad de Lovaina, Bélgica y el doctorado en Ingeniería Mecánica en la FI-UNAM. Ocupa el nivel I en el sistema nacional de investigadores. Durante cuatro años estuvo activo como investigador en proyectos industriales relacionados con la textura cristalográfica en aceros en el Departamento de Metalurgía e Ingeniería de Materiales de la misma Universidad. Su trabajo en México lo inició como experto en microscopía electrónica dentro de la Unidad de Investigación y Asistencia Tecnológica en Materiales (UDIATEM). Posteriormente, combinó estas actividades con una carrera de profesor en la Facultad de Ingeniería. En el ámbito tecnológico se dedica al análisis de falla y la optimización de procesos en la industria metal-mecánica, con un enfoque especial hacia las aleaciones tribológicas dúctiles. Esto ha llevado a una serie de estudios científicos relacionados con las propiedades mecánicas de materiales complejos, los cuales se han publicado en 22 artículos internacionales, 7 en revista internacional y 31 que se han presentado en 30 congresos tanto internacionales como nacionales. 